\title{
TOEPLITZ OPERATORS AND RELATED FUNCTION ALGEBRAS ON CERTAIN PSEUDOCONVEX DOMAINS
}

BY

NICHOLAS P. JEWELL AND STEVEN G. KRANTZ

\begin{abstract}
Toeplitz operators are defined on pseudoconvex domains in $\mathbf{C}^{n}$ and their spectral properties are studied. In addition, the linear space $H^{\infty}+C$ is discussed and is seen to be a closed algebra on a variety of domains.
\end{abstract}

1. Introduction and summary. The Toeplitz operators on the classical Hardy space $H^{2}$ on the unit circle in $\mathbf{C}$ have been the object of much study. They are operators of the form $T_{\varphi} f=P(\varphi f)$ where $\varphi \in L^{\infty}$ and $P$ denotes the projection of $L^{2}$ onto $H^{2}$. An account of this theory can be found in Chapter 7 of R. Douglas' book [8]. Recently attempts have been made to extend this theory to the Hardy spaces of sets in higher dimensions. Notably results have been obtained when the underlying set is the torus in $\mathbf{C}^{n}(n>1)$ (see [7]) and when the set is the unit sphere, $S$, in $\mathbf{C}^{n}$ by L. Coburn [3] and A. M. Davie and N. P. Jewell [5]. The object of the present paper is to extend some of the results on Toeplitz operators acting on $H^{2}(S)$ to Toeplitz operators acting on $H^{2}(\partial D)$ where $D$ is a strongly pseudoconvex domain in $\mathbf{C}^{n}$ (see $\$ \S 2$ and 3) or $D$ is an appropriate weakly pseudoconvex domain in $C^{n}$ (see $\$ \$ 4$ and 5). Some results for the case when $D$ is strongly pseudoconvex have already been obtained in [14], [21], [29], and [30].

In $\$ 2$, for the case $D$ strongly pseudoconvex with some smoothness conditions on the boundary, we establish the spectral inclusion theorem, i.e. the spectrum of $T_{\varphi}$ contains the essential range of $\varphi$. This, in particular, gives the result $\left\|T_{\varphi}\right\|=\|\varphi\|_{\infty}$, extending Theorem 2.1 of [5]. In \$3 we make some remarks about Toeplitz operators with symbol in $H^{\infty}+C$. In $\$ 4$ we establish results analogous to $\S 2$ for the case where $D$ is a totally pseudoconvex domain.

$\$ 5$ contains results on $H^{\infty}+C$ for a class of weakly pseudoconvex domains.

2. The spectral inclusion theorem. In this section we investigate the properties of Toeplitz operators on strongly pseudoconvex domains in $\mathbf{C}^{n}$.

Received by the editors August 21, 1977 and, in revised form, May 8, 1978.

AMS (MOS) subject classifications (1970). Primary 32F15, 47B35.

Key words and phrases. Toeplitz operators, function algebras, pseudoconvex domains. 
Throughout it will be assumed that $n>1$ unless specifically mentioned otherwise. We begin by defining some of the terminology and notation which we will use throughout the paper.

Let $D \subset \subset C^{n}$ have $C^{2}$ boundary, i.e. there is a defining function $\rho$ on a neighborhood $W$ of $\partial D$ so that $\rho$ is of class $C^{2}, D \cap W=\{z \in W: \rho(z)<$ $0\}$, and $\operatorname{grad} \rho \neq 0$ on $\partial D$. We let $\sigma$ denote the surface area measure on $\partial D$. We write $L^{\infty}$ for $L^{\infty}(\sigma), L^{2}$ for $L^{2}(\sigma)$, etc. We use $C$ to denote the space of continuous functions on $\partial D$.

Definition. We define $H^{2}$ to be the closure in $L^{2}$ of the boundary values of functions in $C^{2}(\partial D)$ which extend smoothly to functions holomorphic in $D$. We define $H^{\infty}$ to be the (nontangential) boundary values of the bounded holomorphic functions on $D$.

Definition. Let $D \subset \subset C^{n}$ have $C^{2}$ boundary. We say that $D$ is weakly pseudoconvex if, for each $z \in \partial D$ and each $w \in C^{n}$ satisfying $\sum_{j=1}^{n}\left[\partial \rho(z) / \partial z_{j}\right] w_{j}=0$ one has $\sum_{j, k=1}^{n} \partial^{2} \rho(z) w_{j} \bar{w}_{k} / \partial z_{j} \partial \bar{z}_{k}>0$ for any defining function $\rho$ for $D$. If the quadratic form is positive definite, then we say that $D$ is strongly pseudoconvex.

Remarks. (1) Convex domains are a fortiori pseudoconvex since pseudoconvexity is merely convexity restricted to a subbundle of the complexified tangent bundle.

(2) Let $\rho$ be a defining function for $D$ on $W \supseteq \partial D$, where $D \subset \subset C^{n}$ has $C^{2}$ boundary. For $0<\varepsilon<\varepsilon_{0}$, where $\varepsilon_{0}$ is sufficiently small, we let $D_{e}=(D \backslash$ $W) \cup\{z \in W: \rho(z)<-\varepsilon\}$. Let $\sigma_{\varepsilon}$ be area measure on $\partial D_{\varepsilon}$. Define $\tilde{H}^{2}(D)$ to be

$$
\left\{f: f \text { is holomorphic on } D \text { and } \sup _{0<\varepsilon<\varepsilon_{0}}\left[\int_{\partial D_{e}}|f(z)|^{2} d \sigma_{e}(z)\right]^{1 / 2}<\infty\right\} .
$$

It is shown in [27, pp. 3-4] that the definition is independent of the choice of $\rho$ and $\varepsilon_{0}$. It is unknown whether for any pseudoconvex domain $D$ the function space $\tilde{H}^{2}(D)$ coincides with the space of Poisson extensions of elements of $H^{2}(D)$. Theorem 1 of $\left[27\right.$, p. 5] implies that $H^{2}(D) \subseteq \tilde{H}^{2}(D)$. Conversely, suppose $D$ is starlike with $C^{2}$ boundary and, without loss of generality, suppose that $0 \in D$ is the star point. If $f \in \tilde{H}^{2}(D)$ we define $f_{e}$ by $f_{e}(z)=$ $f(z /(1+\varepsilon))$ for $\varepsilon>0$ small. Then $f \in C^{\infty}(\bar{D})$ and is holomorphic on $D$. Further, $f$ has nontangential boundary values a.e. $(\sigma)$ which we will also denote by $f$ [27, Theorem 1, p. 5]. It follows that $\left.\left.f_{e}\right|_{\partial D} \rightarrow f\right|_{\partial D}$ in $L^{2}(\sigma)$ by [27, pp. 3-4]. So $\tilde{H}^{2}=H^{2}$ for star like domains. The equivalence is known to persist on strongly pseudoconvex domains but the proof relies on much deeper methods which are tangential to the subject of this paper. We refer the reader to [28] for further information on this question. 
Let $D \subset \subset C^{n}$ have $C^{2}$ boundary. If $\varphi \in L^{\infty}(\sigma)$ then we denote by $T_{\varphi}$ the operator on the Hilbert space $H^{2}$ defined by $T_{\varphi} f=P(\varphi \cdot f)$ where $P$ denotes the orthogonal projection of $L^{2}$ on $H^{2}$. The operator $T_{\varphi}$ is called the Toeplitz operator with symbol $\varphi$. For strongly pseudoconvex domains, $T_{\varphi}$ has the integral representation

$$
T_{\varphi} f(z)=\int_{\partial D} \varphi(\zeta) f(\zeta) S(\zeta, z) d \sigma(\zeta)
$$

where $S(\zeta, z)$ is the Szegö kernel (see [27]). Here, for $z \in \partial D, T_{\varphi} f(z)$ is of course the boundary value of $T_{\varphi} f$ at $z$.

For future reference, we note two easily verified identities:

$$
\left(T_{\varphi}\right)^{*}=T_{\bar{\varphi}} \text { and } T_{\varphi} T_{\psi}=T_{\varphi \psi} \quad \text { for } \varphi \in L^{\infty}, \psi \in H^{\infty} \text {. }
$$

On any Hilbert space $H$, we let $I$ denote the identity operator, and $B(H)$ the algebra of bounded linear operators on $H$.

For the remainder of this section we turn our attention to strongly pseudoconvex domains. We first want to prove that $\left\|T_{\varphi}\right\|=\|\varphi\|_{\infty}$. We obtain this result as a corollary of Theorem 2 .

The crux of the proof of the norm equality result for the unit sphere $S$ in $\mathbf{C}^{n}$, given in [5], is the existence, for each point $\zeta \in S$, of a function $f_{\zeta}(z)$ on $\bar{B}=\left\{z \in \mathbf{C}^{n}:|z|<1\right\}$ with the property that $f_{\zeta}(z)$ "peaks" when $z=\zeta$. It is well known that such peaking functions exist for strongly pseudoconvex domains and there are several alternative methods for constructing them. We give [10] as a reference for Theorem 1 since the proof in [10] explicitly shows that the map taking $\zeta \in \partial D$ to $f_{\zeta} \in H^{\infty}$, a function peaking at $\zeta$, is continuous. This is a property which we shall use in the proof of Theorem 2. [10] contains some approximation theorems which also depend on the fact that one can choose peaking functions in a continuous manner.

Definition. Let $D$ be a strongly pseudoconvex domain in $\mathbf{C}^{n}$. Let $\zeta \in \partial D$. A peak function on $D$ at $\zeta$ is a function $f$ such that

(1) $f$ is holomorphic in a neighborhood of $\bar{D}$;

(2) $f(\zeta)=1$;

(3) $|f(z)|<1$ on $\bar{D} \backslash\{\zeta\}$.

THEOREM 1 [10]. Let $D$ be a strongly pseudoconvex domain in $\mathrm{C}^{n}$ with $C^{2}$ boundary. There exist a neighborhood $\hat{D}$ of $\bar{D}$, and a function $f: \partial D \times \hat{D} \rightarrow \mathbf{C}$, such that

(1) $f(\zeta, z)$ is jointly continuous in $\zeta$ and $z$, and holomorphic in $z \in \hat{D}$ for fixed $\zeta$

(2) for each $\zeta \in \partial D, f(\zeta, \cdot)$ is a peak function on $D$ at $\zeta$. The mapping $\zeta \rightarrow f(\zeta, \cdot)$ is continuous from $\partial D$ into $L^{\infty}$ by uniform continuity.

Define a mapping $\eta: L^{\infty} \rightarrow B\left(H^{2}\right)$ by $\eta(\varphi)=T_{\varphi}$. Let $A$ be the closed 
subalgebra of $B\left(H^{2}\right)$ generated by all the Toeplitz operators $T_{\varphi}$ with $\varphi \in L^{\infty}$. Let $J$ be the closed ideal of $A$ generated by all operators of the form $T_{\varphi \psi}-T_{\varphi} T_{\psi}$ where $\varphi, \psi \in L^{\infty}$.

Theorem 2. Let $D$ be a strongly pseudoconvex domain in $\mathbf{C}^{n}$ with $C^{2}$ boundary. The mapping $\eta_{J}$ induced from $L^{\infty}$ to $A / J$ by $\eta$ is an isometric *-isomorphism. In other words, there is a short exact sequence

$$
\{0\} \rightarrow J \rightarrow A \stackrel{\beta}{\rightarrow} L^{\infty} \rightarrow\{0\}
$$

with $\beta\left(T_{\varphi}\right)=\varphi$ for which $\eta$ is an isometric *-linear cross-section.

Proof. The mapping $\eta_{J}$ is clearly linear and contractive. The definition of $J$ shows that it is multiplicative. To complete the proof we show that $\left\|T_{\varphi}+J\right\| \geqslant\|\varphi\|_{\infty}$ for $\varphi \in L^{\infty}$ from which it follows that $\eta_{J}$ is an isometry. To do this, it suffices to show that if $p$ is a noncommuting polynomial in $k$ variables and $\varphi_{1}, \ldots, \varphi_{k} \in L^{\infty}$ then

$$
\left\|p\left(T_{\varphi_{1}}, \ldots, T_{\varphi_{k}}\right)\right\|>\left\|p\left(\varphi_{1}, \ldots, \varphi_{k}\right)\right\|_{\infty} .
$$

Assume that $\left\|p\left(\varphi_{1}, \ldots, \varphi_{k}\right)\right\|_{\infty}=1$. Then there exists $\left(\lambda_{1}, \ldots, \lambda_{k}\right)$ in the joint spectrum of $\left(\varphi_{1}, \ldots, \varphi_{k}\right)$ such that $\left|p\left(\lambda_{1}, \ldots, \lambda_{k}\right)\right|=1$. Let $\varepsilon>0$. Using the fact that $\sum_{j=1}^{k}\left|\varphi_{j}-\lambda_{j}\right|^{2}$ is not bounded below we will show that there exists an $f \in H^{2}$ such that $\|f\|_{2}=1$ and $\int_{\partial D} \Sigma_{j=1}^{k}\left|\varphi_{j}-\lambda_{j}\right|^{2}|f|^{2} d \sigma<\varepsilon^{2}$. The proof is by contradiction. If the assertion is not true, then for all $g \in H^{2}$ with $\|g\|_{2}=1$ we have $\int_{\partial D} \sum_{j=1}^{k}\left|\varphi_{j}-\lambda_{j}\right|^{2}|g|^{2} d \sigma>\varepsilon^{2}$. That is, for all $g \in H^{2}$,

$$
\int_{\partial D} \sum_{j=1}^{k}\left|\varphi_{j}-\lambda_{j}\right|^{2}|g|^{2} d \sigma>\varepsilon^{2} \int_{\partial D}|g|^{2} d \sigma .
$$

Let $\left\{f_{\zeta}: \zeta \in \partial D\right\}$ be a continuous family of peak functions as in Theorem 1 . Put $f_{\zeta}^{m}$ in the inequality (1) where $m \in N$ : we have

$$
\int_{\partial D} \sum_{j=1}^{k}\left|\varphi_{j}(z)-\lambda_{j}\right|^{2}\left|f_{\zeta}(z)\right|^{2 m} d \sigma(z)>\varepsilon^{2} \int_{\partial D}\left|f_{\zeta}(z)\right|^{2 m} d \sigma(z) .
$$

So for any positive $h \in C$,

$$
\begin{gathered}
\int_{\partial D} \int_{\partial D} h(\zeta) \sum_{j=1}^{k}\left|\varphi_{j}(z)-\lambda_{j}\right|^{2}\left|f_{\zeta}(z)\right|^{2 m} d \sigma(z) d \sigma(\zeta) \\
>\varepsilon^{2} \int_{\partial D} \int_{\partial D} h(\zeta)\left|f_{\zeta}(z)\right|^{2 m} d \sigma(z) d \sigma(\zeta) .
\end{gathered}
$$

(Note that the integrals exist since the mapping $\zeta \rightarrow f(\zeta, \cdot)=f_{\zeta}(\cdot)$ is continuous from $\partial D$ into $L^{\infty}$.) Now for any neighborhood $U$ of a fixed $z \in \partial D$ we have 


$$
\frac{\int_{\partial D \backslash U}\left|f_{\zeta}(z)\right|^{2 m} d \sigma(\zeta)}{\int_{\partial D}\left|f_{\zeta}(z)\right|^{2 m} d \sigma(\zeta)} \rightarrow 0 \quad \text { as } m \rightarrow \infty
$$

by the peaking properties of $f_{5}$. Therefore

$$
\frac{\int_{\partial D} h(\zeta)\left|f_{\zeta}(z)\right|^{2 m} d \sigma(\zeta)}{\int_{\partial D}\left|f_{\zeta}(z)\right|^{2 m} d \sigma(\zeta)} \rightarrow h(z) \quad \text { as } m \rightarrow \infty .
$$

Hence, (2) yields

$$
\int_{\partial D} \sum_{j=1}^{k}\left|\varphi_{j}(z)-\lambda_{j}\right|^{2} h(z) d \sigma(z) \geqslant \varepsilon^{2} \int_{\partial D} h(z) d \sigma(z) .
$$

Since this is true for all positive $h \in C$ it follows that $\sum_{j=1}^{k}\left|\varphi_{j}(z)-\lambda_{j}\right|^{2}>\varepsilon^{2}$ almost everywhere on $\partial D$ contradicting the fact that $\sum_{j=1}^{k}\left|\varphi_{j}(z)-\lambda_{j}\right|^{2}$ is not bounded below on $\partial D$. Hence, there exists $f \in H^{2}$ such that $\|f\|_{2}=1$ and $\int_{\partial D} \sum_{j=1}^{k}\left|\varphi_{j}-\lambda_{j}\right|^{2}|f|^{2} d \sigma<\varepsilon^{2}$. So, for each $j,\left\|\left(\varphi_{j}-\lambda_{j}\right) f\right\|_{2}<\varepsilon$ which implies that $\left\|T_{\varphi_{j}} f-\lambda_{j} f\right\|_{2}<\varepsilon$. Then $\left\|p\left(T_{\varphi_{1}}, \ldots, T_{\varphi_{k}}\right) f-p\left(\lambda_{1}, \ldots, \lambda_{k}\right) f\right\|$ can be made arbitrarily small by choosing $\varepsilon$ small enough, whence $\left\|p\left(T_{\varphi_{1}}, \ldots, T_{\varphi_{1}}\right)\right\|$ $>1$. This completes the proof.

Corollary 3. Let $D$ be a strongly pseudoconvex domain in $\mathbf{C}^{n}$ with $C^{2}$ boundary. If $\varphi$ is a function in $L^{\infty}$ such that $T_{\varphi}$ is invertible then $\varphi$ is invertible in $L^{\infty}$.

Notation. The spectrum of a bounded linear operator $T$ on a Hilbert space $H$ will be denoted by $\operatorname{sp}(T)$. We reserve the letter $\sigma$ to denote area measure on $\partial D$.

CoRollary 4. If $\varphi$ is in $L^{\infty}$ then $R(\varphi)=$ essential range of $\varphi=\operatorname{sp}\left(M_{\varphi}\right) \subseteq$ $\operatorname{sp}\left(T_{\varphi}\right)$. (Here $M_{\varphi}$ denotes the operator on $L^{2}$ given by multiplication by $\varphi$.)

Corollary 5. If $\varphi$ is in $L^{\infty}$ then $\left\|T_{\varphi}\right\|=\|\varphi\|_{\infty}$.

For symbols $\varphi \in C$ the norm equality $\left\|T_{\varphi}\right\|=\|\varphi\|_{\infty}$ was essentially obtained by Raeburn [21]. The result is a corollary of [21, 2.2] (which only applied for $n>2$; however, the proof of the necessary part of 2.2 goes through for all $n$ ).

Let $M_{r}(C)$ denote the $C^{*}$-algebra of complex $r \times r$ matrices. Let $L^{\infty}\left(\partial D, M_{r}(C)\right)$ be the functions from $\partial D$ to $M_{r}(C)$ which are essentially bounded. We observe that the analogous result to Theorem 2 for Toeplitz operators, $T_{\varphi}$, with symbol $\varphi$ in $L^{\infty}\left(\partial D, M_{r}(C)\right)$ acting on $H^{2}\left(\partial D, M_{r}(C)\right)$ can be obtained by tensoring the short exact sequence $\{0\} \rightarrow J \rightarrow A \rightarrow L^{\infty} \rightarrow\{0\}$ with $M_{r}(\mathrm{C})$. 
As a further corollary to Theorem 2 we obtain the following sharpening of the spectral inclusion Theorem in the case $n>2$.

Corollary 6. Let $n>2$. If $\varphi \in L^{\infty}$ then $\Re(\varphi) \subseteq \operatorname{sp}_{e}\left(T_{\varphi}\right) \equiv$ essential spectrum of $T_{\varphi}$.

Proof. By a result of Raeburn [21, 2.2], $J$ contains all the compact operators. Now $\operatorname{sp}_{e}\left(T_{\varphi}\right)$ is the spectrum of the coset of $T_{\varphi}$ in $A / \mathcal{K}$ where $\mathcal{K}$ is the ideal of all compact operators on $H^{2}$. Thus $\operatorname{sp}_{e}\left(T_{\varphi}\right)$ contains the spectrum of $T_{\varphi}$ in $A / J$ and hence, by Theorem 2, contains $R(\varphi)$.

As in [5], where $D=B=$ the unit ball in $C^{n}$, we will now identify the spectra, $\operatorname{sp}\left(T_{\varphi}\right)$, when $\varphi \in H^{\infty}$.

Proposition 7. Let $n>2$. If $\varphi \in H^{\infty}$ then $\operatorname{sp}_{e}\left(T_{\varphi}\right)$ (and so also $\operatorname{sp}\left(T_{\varphi}\right)$ ) coincides with the spectrum of $\varphi$ in $H^{\infty}$.

Proof. We have to show that if $T_{\varphi}$ is Fredholm then $\varphi$ is invertible in $H^{\infty}$. Corollary 6 shows that $\varphi$ is invertible in $L^{\infty}$. Then $T_{\varphi^{-1}} T_{\varphi}=I$ so that the operator $H_{\varphi^{-1}} ; H^{2} \rightarrow L^{2}$ defined by $H_{\varphi^{-1}} f=\varphi^{-1} f-T_{\varphi^{-1}} f$ has finite rank. Hence there is an integer $m$ and complex numbers $a_{1}, \ldots, a_{m}$, not all zero, so that $H_{\varphi^{-1}} f=0$ where $f(z)=\sum_{k=0}^{m} a_{k} z_{1}^{k}$. So $\varphi^{-1} f \in H^{2}$. But since $f \in H^{\infty}$ we have $\varphi^{-1} f \in H^{\infty}$. Now if $g \in L^{\infty}$ and $g(z)\left(z_{1}-\lambda\right) \in H^{\infty}$ then the harmonic extension of $g$ to $D$ is bounded and analytic except possibly where $z_{1}=\lambda$. By the Riemann continuation theorem (see $[19$, p. 51]) we can remove any such singularities of $g$ and so $g$ is holomorphic on $D$. Thus $\varphi^{-1} f \in H^{\infty}$ implies that $\varphi^{-1} \in H^{\infty}$, i.e. $\varphi$ is invertible in $H^{\infty}$.

The algebra $H^{\infty}+C$. We denote by $H^{\infty}+C$ the set of all functions $f \in L^{\infty}$ which can be expressed in the form $f=u+v$ where $u \in H^{\infty}$ and $v \in C$. When $D=B$, Rudin [26] showed that $H^{\infty}+C$ is a closed subalgebra of $L^{\infty}$. For general strongly pseudoconvex domains $D$ with $C^{2}$ boundary, Aytuna and Chollet [1] extended Rudin's result to show that $H^{\infty}+C$ is a closed subalgebra of $L^{\infty}$ in this case also. For interest, given that $H^{\infty}+C$ is closed, we give an alternative proof of the fact that it is also an algebra using ideas contained in [18].

Proposition 8. Let $D$ be a strongly pseudoconvex domain with $C^{2}$ boundary. Then $H^{\infty}+C$ is a closed subalgebra of $L^{\infty}$.

Proof. The fact that $H^{\infty}(D)+C(\bar{D})$ and $H^{\infty}+C$ are closed follows from a theorem of Gamelin and Garnett on rational approximation as shown in [1]. $\left[H^{\infty}(D)\right.$ is the algebra of all bounded holomorphic functions on $D$ and $C(\bar{D})$, the algebra of all continuous functions on $\bar{D}$.] Let $C^{\infty}(D)$ denote the collection of smooth functions on $D$. Put $\mathscr{B}=\left\{\varphi \in C^{\infty}(D): \varphi, \bar{\partial} \varphi\right.$ are bounded $\}. \mathscr{B}$ is clearly an algebra. Also, for $\varphi \in \mathscr{B}, \bar{\partial} \varphi$ is a smooth bounded 
$\bar{\partial}$-closed $(0,1)$ form on $D$ and so, by [15, pp. 342-345], there exists $u \in$ $C^{\infty}(D) \cap C(\bar{D})$ such that $\bar{\partial} u=\bar{\partial} \varphi$. Thus $\varphi-u \in H^{\infty}(D)$ and hence $\varphi \in$ $H^{\infty}(D)+C(\bar{D})$. Thus $\mathscr{B} \subseteq H^{\infty}(D)+C(\bar{D})$. Clearly $\mathscr{B}$ contains $C^{\infty}(\bar{D})$, the set of smooth functions on $D$ which extend smoothly to $\bar{D}$. But $C^{\infty}(\bar{D})$ is dense in $C(\bar{D})$. Hence $\mathscr{B}$ is a dense subalgebra of $H^{\infty}(D)+C(\bar{D})$. The fact that $H^{\infty}(D)+C(\bar{D})$ is closed then shows that $H^{\infty}(D)+C(\bar{D})$ is a closed algebra. Let $f \in H^{\infty}(D)$. For every $z \in \partial D$ let $\nu_{z}$ be the outward normal to $\partial D$ at $z$ and put $f^{*}(z)=\lim _{\varepsilon \rightarrow 0} f\left(z-\varepsilon \nu_{z}\right)$. Such a limit exists almost everywhere on $\partial D$ (see [27]). In fact the map $f \rightarrow f^{*}$ gives an isometric isomorphism of $H^{\infty}(D)$ onto $H^{\infty}$ (see [1], [27, p. 5]). From this we deduce that $H^{\infty}(D)+$ $C(\bar{D})$ being an algebra implies that $H^{\infty}+C$ is an algebra. This completes the proof of the proposition.

For the case $n>2$ Raeburn [21, 2.2] showed that $T_{\varphi} T_{\psi}-T_{\varphi \psi}$ is compact for every $\varphi, \psi \in C$. Hence the commutator ideal of the closed algebra generated by $\left\{T_{\varphi}: \varphi \in C\right\}$ in $B\left(H^{2}\right)$ is precisely the ideal of all compact operators on $H^{2}$, and coincides with the closed ideal generated by $\left\{T_{\varphi \psi}-\right.$ $T_{\varphi} T_{\psi}: \varphi, \psi \in C$ \}. Also, for $n>2$, Raeburn [21, Theorem 1.2] showed that if $\varphi \in C$ then $M_{\varphi}-P M_{\varphi}: H^{2} \rightarrow L^{2}$ is compact. This implies that $T_{\varphi}$ commutes modulo the compact operators with any Toeplitz operator. Hence the commutator ideal of the closed algebra generated by $\left\{T_{\varphi}: \varphi \in H^{\infty}+C\right\}$ is also just the ideal of all compact operators on $\mathrm{H}^{2}$, and coincides with the closed ideal generated by $\left\{T_{\varphi \psi}-T_{\varphi} T_{\psi}: \varphi, \psi \in H^{\infty}+C\right\}$. It then follows from Theorem 5 that the closed algebra generated by $\left\{T_{\varphi}: \varphi \in H^{\infty}+C\right\}$ is precisely the set of operators of the form $T_{\varphi}+K$ where $\varphi \in H^{\infty}+C$ and $K$ is a compact operator on $H^{2}$. This extends Theorem 7.29 of [8] and results in [5] to strongly pseudoconvex domains in $\mathbf{C}^{n}, n>2$, with $C^{2}$ boundary. The problem with the case $n=2$ in the above remarks (and in Corollary 6 and Proposition 7) is that the Neumann Theory for the $\bar{\partial}_{b}$ operator does not go through in the same way for $n=2$ as it does for $n>2$. (For $n=2, \bar{\partial}_{b}$ is essentially the $H$. Lewy unsolvable operator.) It is possible that these obstructions can be circumvented with new techniques.

Similar results for Toeplitz operators on $H^{2}(D)$ (the set of square integrable functions with respect to volume measure on $D$ which are holomorphic on $D$ ) have been obtained by Janas [14] and Raeburn [21]. The same extensions obtained above for Toeplitz operators with symbols in $H^{\infty}(D)+C(\bar{D})$ can be deduced from their results. For symbols $\varphi \in C(\bar{D})$ the norm equality $\left\|T_{\varphi}\right\|=\|\varphi\|_{\infty}$ was obtained by Janas [14] for strongly pseudoconvex domains $D$ in $C^{n}$ with $C^{2}$ boundary under the additional hypothesis that $A(D)=$ $P(D)$. Here $A(D)$ denotes the algebra of all functions continuous on $\bar{D}$ and holomorphic on $D$, and $P(D)$ denotes the algebra of all functions on $\bar{D}$ which can be uniformly approximated by holomorphic polynomials on $\bar{D}$. Indepen- 
dently Raeburn [21] and Yabuta [30] observed that the assumption $A(D)=$ $P(D)$ could be removed. It is clear that the equality $\left\|T_{\varphi}\right\|=\|\varphi\|_{\infty}$ cannot hold for every essentially bounded function $\varphi$ on $D$. It should be noted here that many of the ideas concerning Toeplitz operators on strongly pseudoconvex domains originated in Venugopalkrishna's paper [29].

Finally note that if $\varphi \in H^{\infty}$ and $\varphi$ is invertible in $H^{\infty}+C$ then $\varphi^{-1} \in$ $H^{\infty}$. (This follows by using the Poisson integral and then applying Hartog's theorem [13, p. 30].) Hence if $\varphi \in H^{\infty}+C$ and $\varphi$ invertible in $L^{\infty}$ implies $\varphi^{-1} \in H^{\infty}+C$ we would then have that any inner function on $\partial D$ is constant $\left(\varphi \in H^{\infty}\right.$ is inner if $|\varphi|=1$ a.e. on $\left.\partial D\right)$. It is unknown whether or not there are nonconstant inner functions on $B$, the unit ball in $\mathbf{C}^{n}$; for a discussion of this and related problems see [5].

4. Results on weakly pseudoconvex domains. In this section we investigate extensions of the principal results of $\$ 2$ to weakly pseudoconvex domains.

Pseudoconvex domains are domains of holomorphy and are therefore the natural arena for complex function theory. However, it is not known for every pseudoconvex domain which boundary points are peak points. We therefore impose additional hypotheses which will enable us to make the necessary constructions.

Definition. Let $D \subset \subset C^{n}$ have $C^{1}$ boundary. If $P \in \partial D$, let $T_{P}$ denote the $(2 n-1)$ dimensional real tangent space to $\partial D$ at $P$. The holomorphic tangent space $\mathcal{H}_{P}$ is defined to be $T_{P} \cap \sqrt{-1} T_{P}$.

Definition. Let $D \subset C^{n}$ have $C^{2}$ boundary. Let $\rho$ be a $C^{2}$ defining function for $D$ on a neighborhood $W \supseteq \partial D$. We say that $D$ is totally pseudoconvex at $P \in \partial D$ if there is a holomorphic change of coordinates $w=w(z)$ in a neighborhood $U$ of $P$ so that

$$
w(\bar{D} \cap U) \cap \mathcal{H}_{w(P)}(\partial(w(D \cap U)))=\{w(P)\} .
$$

The domain $D$ is said to be totally pseudoconvex if every $P \in \partial D$ is totally pseudoconvex.

REMARK. Of course, totally pseudoconvex domains are pseudoconvex. A routine application of the implicit function theorem, carried out in [22, p. 6], shows that the preceding definition is equivalent with the following more classical one of Behnke and Thullen:

The domain $D$ is totally pseudoconvex at $P \in \partial D$ if there is a nonsingular analytic hypersurface $M$ in a neighborhood $U$ of $P$ so that $M \cap \bar{D} \cap U=$ $\{P\}$.

That a totally pseudoconvex domain in $\mathbf{C}^{n}$ has local holomorphic peaking functions is proved by Range [22, Corollary 1.5]. The proof is so brief that we include it. 
Proposition 9. Let $D \subseteq C^{n}$ be totally pseudoconvex at $P \in \partial D$. There is a neighborhood $U$ of $P$, a neighborhood $\Omega$ of $\bar{D} \cap U \sim\{P\}$, and a biholomorphic map $G: \Omega \rightarrow G(\Omega) \subseteq \mathbf{C}^{n}$ so that

(1) $G$ extends continuously to $P$ and $G(P)=0$.

(2) $G(\bar{D} \cap U \sim\{P\}) \subseteq\left\{w \in \mathbf{C}^{n}: \operatorname{Re} w_{n}>0\right\}$.

Proof. Let notation be as in the definition of totally pseudoconvex. Write $w=F(z)$. By a linear transformation of coordinates, we may suppose that $w(P)=0$ and that $\mathcal{K}_{w(P)}=\left\{w: w_{n}=0\right\}$. Let $r$ be a defining function for $D$. Then $\rho=r \circ F^{-1}$ is a defining function for $F(D \cap U)$ near 0 and $\partial_{w} \rho(0)=$ $K d w_{n}$, for some nonzero $K$. After a normalization, we may suppose that $K=1$. Write $w=\left(w_{1}, \ldots, w_{n}\right)=\left(w^{\prime}, w_{n}\right)$. By hypothesis, there is a $c>0$ so that $\rho\left(w^{\prime}, 0\right)>0$ for $0<\left|w^{\prime}\right|<c$. Using Taylor's theorem, we see that for $|w|<c, w^{\prime} \neq 0$, we have

$$
\begin{aligned}
\rho(w) & =\rho\left(w^{\prime}, 0\right)+2 \operatorname{Re}\left(\frac{\partial \rho}{\partial w_{n}}\left(w^{\prime}, 0\right) \cdot w_{n}\right)+o\left(\left|w_{n}\right|\right) \\
& >\operatorname{Re} 1 \cdot w_{n}+o(1) w_{n} .
\end{aligned}
$$

Shrinking $U$ if necessary, we may suppose that $o(1) \cdot\left|w_{n}\right|<1 / 2\left|\operatorname{Re} w_{n}\right|+$ $1 / 2\left|\operatorname{Im} w_{n}\right|$. Thus if $w$ is any point at which $\rho(w)<0$ we have

$$
0>2 \rho(w)>2 \operatorname{Re} w_{n}-\left|\operatorname{Re} w_{n}\right|-\left|\operatorname{Im} w_{n}\right| \text { or }-\operatorname{Re} w_{n}>-\left|\operatorname{Im} w_{n}\right| .
$$

It follows that on $F(\bar{D} \cap U \backslash\{P\}),-w_{n}$ omits the nonpositive real axis $\mathbf{R}^{-}$. If one chooses that branch of the square root on $\mathbf{C} \backslash \mathbf{R}^{-}$which satisfies $\sqrt{1}=1$ then $\sqrt{-w_{n}}$ is holomorphic on $\mathbf{C} \backslash \mathbf{R}^{-}$and

$$
\operatorname{Re} \sqrt{-w_{n}}>0 \text { for } w \in F(\bar{D} \cap U \sim\{P\}) \text {. }
$$

This inequality persists for $w \in F(\Omega)$ where $\Omega$ is some neighborhood of $\bar{D} \cap U \backslash\{P\}$. If we let $S(w)=\left(w^{\prime}, \sqrt{-w_{n}}\right)$ then $G=S \circ F$ satisfies all the conclusions of the proposition.

Corollary 10. With notation as in Proposition 9, $P$ is a peak point for the algebra $A(D \cap U)$.

Proof. The function $h(z)=\exp (-G(z))$ is in $A(D \cap U)$ and peaks at $P$.

Corollary 11. Let $D \subset \subset C^{n}$ have $C^{\infty}$ boundary, be pseudoconvex, and be totally pseudoconvex at $P \in \partial D$. Then $P$ is a peak point for the algebra $A(D)$.

Proof. By a deep theorem of Kohn [16, p. 283], if $f=\sum_{j=1}^{n} f_{j} d \bar{z}_{j}$ is a $\bar{\partial}$ closed $(0,1)$ from satisfying $f_{j} \in C^{\infty}(\bar{D})$ for each $j$, then there is a $u \in$ $C^{\infty}(\bar{D})$ such that $\bar{\partial} u=f$. Using this fact and a standard construction [11, p. 406], one can "extend" the peaking function of Corollary 10 to all of $\bar{D}$.

REMARK. An examination of Kohn's proof reveals that $C^{\infty}$ boundary is not 
necessary in many cases to obtain his result (with an appropriately weakened conclusion). However, Kohn's techniques work so well because they suppress boundary behavior. Thus a careful study of minimal smoothness hypotheses for Corollary 11 would far exceed the scope of this paper. We will therefore let all domains in this section have $C^{\infty}$ boundary.

An important feature of Theorem 1 is that on strongly pseudoconvex domains one can construct peaking functions which vary continuously with the peak point. Whether this can be done on a domain which is totally pseudoconvex at each point of $\partial D$ or, more generally, on any pseudoconvex domain, is unknown to us. ${ }^{1}$ However, it is sufficient for our applications to know that the function $f: \partial D \times \bar{D} \rightarrow \mathbf{C}$ constructed in Theorem 1 is measurable on $\partial D \times \partial D$. That this is true in general follows from the remarkable von Neumann selection theorem:

Theorem 12 (VON NeUMANN [20, P. 448]). Let $S$ be a complete and separable metric space and $\mathcal{P}$ an analytic (in the sense of Souslin and Lusin) subset of $S$. Let $m \in\{1,2, \ldots\}$ and let $G: \stackrel{P}{\rightarrow} \rightarrow \mathbf{R}^{m}$ be continuous. Let $K=G(\mathcal{P})$. Then

(i) $K$ is measurable.

(ii) There is a function $g: K \rightarrow \mathscr{P}$ such that $g$ is measurable (under the usual Lebesgue measure on $K$ ) and $G(g(k))=k$ for all $k \in K$.

REMARK. In fact von Neumann proves a stronger result for a more restricted collection of mappings. However, his elementary (but very elegant) techniques readily apply to the situation in Theorem 12 . We would like to thank A. Kechris and D. Martin for bringing these results to our attention.

Our application of von Neumann's theorem is almost immediate. Let $A(\partial D)$ denote the elements of $A(D)$ restricted to $\partial D$. Let $D \subset \subset \mathrm{C}^{n}$ have the property that each $P \in \partial D$ is a peak point for the algebra $A(\partial D)$. Let $\mathscr{P} \subseteq \partial D \times A(\partial D)$ denote the set of ordered pairs $(P, \varphi)$ such that $\varphi(P)=1$ and $|\varphi(z)|<1$ for $z \in D \backslash\{P\}$. Let $E \subset \partial D$ be a countable dense set. Equip $A(\partial D)$ with the uniform topology and $\partial D \times A(D)$ with the product topology. Then the identity

$$
\begin{aligned}
\mathscr{P}= & \bigcap_{n} \bigcup_{x \in E} \bigcup_{n>m}\{\zeta \in \partial D:|\zeta-x|<1 / m\} \\
& \times\{\varphi \in A(\partial D):\|\varphi\|<1+1 / m, \operatorname{Re} \varphi(x)>1-1 / n, \text { and } \\
& |\varphi(z)|<1-2 / n \text { for all } z \in \partial D \text { such that }|z-x|>1 / m\}
\end{aligned}
$$

demonstrates that $\mathscr{P}$ is a $G_{\delta}$ in $\partial D \times A(\partial D)$ and hence is Borel (and thus analytic). We may consider the map $G=\pi_{1}: \mathscr{P} \rightarrow \partial D$ consisting of projection on the first coordinate. Notice that $\mathcal{P} \subseteq \partial D \times A(\partial D) \subset \partial D \times C(\partial D)$

\footnotetext{
'It has recently been proved by J. E. Fornaess and the second author that peaking functions can be chosen in a continuous fashion in any function algebra on any compact metric space $X$.
} 
which is separable. Since $\partial D$ is locally diffeomorphic with $\mathbf{R}^{2 n-1}$, the von Neumann selection theorem guarantees the existence of a right inverse $g$ for $G$ which has the property that $g: \partial D \rightarrow \mathscr{P}$ is Lebesgue measurable. Since elements of $A(\partial D)$ are continuous on $\bar{D}$, and since $A(\partial D)$ is equipped with the uniform topology, it follows that

$$
f: \partial D \times \partial D \rightarrow \mathbf{C}
$$

given by $f(\zeta, z)=\left[\pi_{2}(g(\zeta))\right](z)$ is product measurable. Here $\pi_{2}$ is projection on the second coordinate.

The preceding arguments apply to any domain on which one has peaking functions at each boundary point. We now state the precise result which will be used in this section.

Theorem 13. Suppose $D \subset \subset C^{n}$ has $C^{\infty}$ boundary and that $D$ is totally pseudoconvex. Then there is a map $f: \partial D \times \partial D \rightarrow \mathbf{C}$ satisfying

(i) $f$ is product measurable with respect to the measure $\sigma \times \sigma$ ( $\sigma$ is Hausdorff $2 n-1$ dimensional measure, or area measure, on $\partial D)$;

(ii) for each fixed $\zeta \in \partial D, f(\zeta, \cdot) \in A(\partial D)$;

(iii) for each $\zeta \in \partial D, f(\zeta, \zeta)=1$ and $|f(\zeta, z)|<1$ for all $z \in \partial D \backslash\{\zeta\}$.

If we define Toeplitz operators on general $D \subset \subset C^{n}$ just as in $\$ 2$ we then have

THEOREM 14. If $D \subset \subset C^{n}$ is totally pseudoconvex with $\mathbf{C}^{\infty}$ boundary then the conclusions of Theorem 2, Corollary 3, Corollary 4, and Corollary 5 hold on D.

Proof. Theorem 13 guarantees the existence of measurably varying peaking functions for $A(D)$. Therefore the proofs are identical to those in $\$ 2$.

REMARK. It follows from a theorem of Range [22, Corollary 1.13] that a bounded convex domain in $\mathbf{C}^{n}$ with real analytic boundary is totally pseudoconvex (he actually proves much more). Therefore Theorem 14 applies to bounded convex domains with real analytic boundary.

REMARK. We are unable to prove Corollary 6 for totally pseudoconvex domains by existing techniques because an appropriate version of Raeburn's theorem is unavailable. More precisely, the construction of a Neumann operator for totally pseudoconvex domains is a deep problem. Thus if $P$ : $L^{2} \rightarrow H^{2}$ is the projection of $L^{2}$ on $H^{2}$ and if $\varphi \in C$, we do not know whether $(I-P) M_{\varphi}$ is a compact operator. The recent advances of Kohn [17] on subelliptic estimates for the $\bar{\partial}$ operator on weakly pseudoconvex domains may shed some light on this problem.

5 Further results on $H^{\infty}+C$. Let $D \subset \subset C^{n}$ have $C^{2}$ boundary. We will continue to use the notation $H^{\infty}$ to signify boundary values of bounded holomorphic functions on $D$. We will identify elements of $H^{\infty}$ with their 
Poisson integrals on $D$, and we do likewise with elements of $C$.

If $f=\Sigma f_{j} d \bar{z}_{j}$ is a $(0,1)$ form, we let $\|f\|_{\infty}=\Sigma\left\|f_{j}\right\|_{\infty}$. We let $K_{0,1}^{\infty}$ denote the $\bar{\partial}$ closed $(0,1)$ forms with bounded, $C^{1}$ coefficients.

We will make use of the following result of [23, p. 32], [24]:

TheOREM 15 (RANGE). Let $D \subset \subset C^{2}$ be convex and have real analytic boundary. There are constants $M, \alpha>0$ so that if $f \in K_{0,1}^{\infty}(D)$ then there is a $u \in C^{1}(D)$ satisfying $\bar{\partial} u=f$ on $D$ and

$$
\sup _{z, z^{\prime} \in D}\left|u(z)-u\left(z^{\prime}\right)\right|<M\|f\|_{\infty}\left|z-z^{\prime}\right|^{\alpha} .
$$

Further, the map $T: f \rightarrow u$ is linear.

REMARK. The last sentence is not stated explicitly in [23]. However, linearity is an automatic feature of the Henkin construction which Range uses.

COROLlary 16. With hypotheses as in the theorem, there is an $M^{\prime}>0$ and a linear map $S: K_{0,1}^{\infty}(D) \rightarrow C^{1}(D)$ so that $\bar{\partial} S f=f$,

$$
\sup _{z, z^{\prime} \in D}\left|S f(z)-S f\left(z^{\prime}\right)\right|<M\|f\|_{\infty}\left|z-z^{\prime}\right|^{\alpha}
$$

and

$$
\|S f\|_{\infty}<M^{\prime}\|f\|_{\infty}
$$

Proof. Fix some $P \in D$ and define $S f(z)=T f(z)-T f(P)$. The corollary is immediate with $M^{\prime}=M \cdot(\operatorname{diam} D)^{\alpha}$.

Corollary 17. If $f \in K_{0,1}^{\infty}(D)$ then both $T f$ and $S f$ extend continuously to $\bar{D}$.

COROLlaRY 18. If $\left\{w_{n}\right\} \subseteq K_{0,1}^{\infty}(D)$ and if $w_{n}$ converges weak * to 0 in the space of $(0,1)$ forms with $L^{\infty}$ coefficients, then $S\left(w_{n}\right)$ converges uniformly to 0 in $C(\bar{D})$.

Proof. Following the Henkin construction, the function $S f$ is given by

$$
S f(z)=\sum_{i=1}^{n} \int_{\zeta \in \partial D} f_{i}(\zeta) K_{i}(z, \zeta) d \sigma(\zeta) \quad \text { where } f=\sum f_{i} d \bar{z}_{i}
$$

and $K: D \times \partial D \rightarrow C$ is a function which, for each fixed $z \in D$, satisfies $K_{i}(z, \cdot) \in L^{1}(\partial D)$. In the works [23], [24], the method of [25] is used to prove Theorem 15. That is, one shows that

$$
\int_{\partial D}\left|K_{i}(z, \zeta)-K_{i}\left(z^{\prime}, \zeta\right)\right| d \sigma(\zeta)<(M / n)\left|z-z^{\prime}\right|^{\alpha} .
$$

Let $L_{i}: D \times \partial D \rightarrow \mathrm{C}$ be given by $L_{i}(z, \zeta)=K_{i}(z, \zeta)-K_{i}(P, \zeta), \quad i=$ $1, \ldots, n$, with $P$ as in the proof of Corollary 16. Then 


$$
\begin{aligned}
S w_{n}(z) & =\sum_{i=1}^{n} \int_{\zeta \in \partial D} L_{i}(z, \zeta) f(\zeta) d \sigma(\zeta) \text { and } \\
\left\|L_{i}(z, \cdot)\right\|_{L^{\prime}(\partial D)} & \leqslant(M / n)|z-P|^{\alpha}<(M / n)(\operatorname{diam} D)^{\alpha}
\end{aligned}
$$

by line (1). Finally, $S w_{n}$ extends continuously to $\bar{D}$ for each $n$ by Corollary 15 . Line (2) and the weak * convergence of $\left\{w_{n}\right\}$ implies that $S w_{n}(z)$ converges pointwise to 0 for each $z \in D$. This fact coupled with the Lipschitz estimates for $S$ and the compactness of $\bar{D}$ implies that $S w_{n}$ converges uniformly to 0 on $\bar{D}$.

Definition. Let $D \subset \subset C^{n}$ have $C^{2}$ boundary. Let $\mu$ be a finite Borel measure on $\partial D$ and $A(D)$ be as usual. Following Henkin [12], we say that $\mu$ is an $A$ measure if

$$
\lim _{n \rightarrow \infty} \int_{\partial D} f_{n} d \mu=0
$$

whenever $\left\{f_{n}\right\} \subseteq A(D)$ is a bounded sequence which converges pointwise to 0 .

Definition. Let $D, \mu, A(D)$ be as in the preceding definition. We may think of $A(D)$ as a subspace of $L^{\infty}(\mu)$. We let $H^{\infty}(\mu)$ denote the weak * closure of $A(D)$ in $L^{\infty}(\mu)$. We define $H^{\infty}(\mu)$ likewise when $\mu$ is a finite Borel measure on $\bar{D}$.

Theorem 19. Let $D \subset \subset C^{n}$ have $C^{2}$ boundary. Suppose that there is a linear operator $S: K_{0,1}^{\infty}(D) \rightarrow C^{1}(D) \cap C(\bar{D})$ satisfying

(1) $\bar{\partial} S f=f$.

(2) If $\left\{w_{n}\right\} \subseteq K_{(0,1)}^{\infty}(D)$ converges weak $*$ to 0 in the space of $(0,1)$ forms with $L^{\infty}$ coefficients, then $S\left(w_{n}\right)$ converges uniformly to 0 in $C(\bar{D})$.

Suppose further that

(3) If $f \in H^{\infty}$ there is a bounded sequence $f_{n} \in A(D)$ which converges pointwise to $f$ on $D$ and a.e. to $f$ on $\partial D$.

Then

(4) Any finite Borel measure on $\partial D$ which is absolutely continuous with respect to an $A$ measure is itself an $A$ measure;

(5) if $\mu$ is a finite Borel measure on $\bar{D}$ such that $\mu \mid \partial D$ is an $A$ measure and if $v$ denotes volume measure on $D$ then the restriction map $H^{\infty}(|\mu|+v) \rightarrow$ $H^{\infty}(v)$ is an isometric isomorphism;

(6) if $\mu$ is an $A$ measure on $\partial D$ and if $\sigma$ denotes area measure on $\partial D$ then the restriction map $H^{\infty}(|\mu|+v) \rightarrow H^{\infty}(v)$ is an isometric isomorphism.

Proof. These are two of the principal results of [4] for strongly pseudoconvex domains $D$. However it is a beautiful feature of that paper that strong pseudoconvexity is used only to guarantee (1), (2), and (3).

We note in passing that Cole and Range [4] work on a complex manifold 
so they must select a Kähler metric. Since our $D$ is in $C^{n}$, we use the ordinary Euclidean metric.

Now we state the principal result of this section:

THEOREM 20. Let $D \subset \subset C^{2}$ be convex with real analytic boundary. Then $H^{\infty}+C$ and $H^{\infty}(D)+C(\bar{D})$ are closed.

Proof. We wish to apply the method of Aytuna and Chollet in [1]. For this, one needs to know that

(1) $A(D)$ has peaking functions (not necessarily varying continuously) at each $P \in \partial D$.

(2) For each $f \in H^{\infty}$ there is a bounded sequence in $A(D)$ which converges pointwise to $f$ on $D$ and pointwise a.e. to $f$ on $\partial D$.

(3) The conclusions (4), (5), and (6) of Theorem 19 hold on $D$.

That (1) holds in the present circumstances is of course Theorem 13 and the remark following Theorem 14. That (2) is true follows by a dilation argument. That (3) is true is the content of Theorem 15 through Theorem 19.

TheOREM 21. Let $D \subset \subset C^{2}$ be convex with real analytic boundary. Then $H^{\infty}(D)+C(\bar{D})$ and $H^{\infty}+C$ are closed algebras.

Proof. In order to repeat the proof of Proposition 8, we need only know that if $f \in K_{0,1}^{\infty}(D)$ then there is a $u \in C^{1}(D) \cap C(\bar{D})$ with $\bar{\partial} u=f$. This is Corollary 17.

REMARK. (1) If $m=\left(m_{1}, \ldots, m_{n}\right)$ is a tuple of positive integers we let $B_{m}=\left\{z \in C^{n}: \Sigma\left|z_{i}\right|^{2 m_{i}}<1\right\}$. Then $B_{m}$ is bounded, convex, and has real analytic boundary. For these domains Range has been able to overcome the technical obstructions to Theorem 15 when $n>2$ (private communication). Therefore it follows as above that $H^{\infty}\left(B_{m}\right)+C\left(\bar{B}_{m}\right)$ and $H^{\infty}\left(\partial B_{m}\right)+C\left(\partial B_{m}\right)$ are closed algebras.

(2) There is an abstract uniform algebra theorem in [9] which can be used to prove Proposition 8, Theorem 20 and Theorem 21. One uses the integral kernel of Henkin-Ramirez for the strongly pseudoconvex case and the kernel of Range for the weakly pseudoconvex case to construct the necessary "Remainder Operators" which are needed in [9]. What we have done in the proof of Theorems 20 and 21 is to cast the same calculations in a different form.

Acknowledgement. The first author would like to express his thanks to the Commonwealth Fund of New York which has supported this research in the form of a Harkness Fellowship.

The second author is grateful to the National Science Foundation for partial support from NSF Grant No. MPS-74-7035. He is also grateful to the Université de Paris-Sud for its hospitality during a part of this work. 


\section{REFERENCES}

1. A. Aytuna and A.-M. Chollet, Une extension d'un resultat de W. Rudin, Bull. Soc. Math. France 104 (1976), 383-388.

2. E. Bedford and J. E. Fornaess, Domains with pseudoconvex neighborhood systems, Invent. Math. 47 (1978), 1-27.

3. L. A. Coburn, Singular integral operators and Toeplitz operators on odd spheres, Indiana Univ. Math. J. 23 (1973), 433-439.

4. B. Cole and R. M. Range, A-measures on complex manifolds and some applications, J. Functional Analysis 11 (1973), 393-400.

5. A. M. Davie and N. P. Jewell, Toeplitz operators in several complex variables, J. Functional Analysis 26 (1977), 356-368.

6. K. Diederich and J. E. Fornaess, A strange bounded domain of holomorphy, Bull. Amer. Math. Soc. 207 (1975), 219-240.

7. R. M. Douglas, Banach algebra techniques in the theory of Toeplitz operators, CBMS Regional Conf. Ser. in Math. no. 15, Amer. Math. Soc., Providence, R.I., 1973.

8. __ Banach algebra techniques in operator theory, Academic Press, New York, 1972.

9. T. W. Gamelin, Rational approximation theory, Lecture notes, U.C.L.A., 1975.

10. I. Graham, Boundary behavior of the Caratheodory and Kobayashi metrics on strongly pseudoconvex domains in Cn, Trans. Amer. Math. Soc. 207 (1975), 219-240.

11. M. Hakim and N. Sibony, Quelques conditions pour l'existence de fonctions pics dans les domaines pseudoconvexes, Duke Math. J. 44 (1977), 399-406.

12. G. M. Henkin, Integral representations of functions holomorphic in strictly pseudoconvex domains and some applications, Math. USSR Sb. 7 (1969), 597-616.

13. L. Hörmander, An introduction to complex analysis in several complex variables, Van Nostrand, New York, 1966.

14. J. Janas, Toeplitz operators related to certain domains in $\mathbf{C}^{n}$, Studia Math. 54 (1975), 73-79.

15. N. Kerzman, Hölder and $L^{p}$ estimates for solutions to $\bar{\partial} u=f$ in strongly pseudoconvex domains, Comm. Pure Appl. Math. 24 (1971), 301-379.

16. J. J. Kohn, Global regularity for $\bar{\partial}$ on weakly pseudoconvex manifolds, Trans. Amer. Math. Soc. 181 (1973), 273-292.

17. Sufficient conditions for subellipticity on weakly pseudoconvex domains, Proc. Nat. Acad. Sci. U.S.A. 94 (1977), 2214-2216.

18. G. McDonald, Fredholm properties of a class of Toeplitz operators on the ball, Indiana J. Math. 26 (1977), 567-576.

19. R. Narasimhan, Several complex variables, Univ. of Chicago Press, Chicago, Ill., 1971.

20. J. von Neumann, On rings of operators, reduction theory, Ann. of Math. (2) 50 (1949), 401-485.

21. I. Raeburn, On Toeplitz operators associated with strongly pseudoconvex domains, Studia Math. 63 (1979), 253-258.

22. R. M. Range, The Carathéodory metric and holomorphic maps on a class of weakly pseudoconvex domains (preprint).

23. _ Hölder estimates for $\bar{\partial}$ on convex domains in $\mathbf{C}^{2}$ with real analytic boundary, Proc. Sympos. Pure Math., vol. 30, Amer. Math. Soc., Providence, R.I., 1977, pp. 31-33.

24. __ On Hölder estimates for $\partial u=f$ on weakly pseudoconvex domains (preprint).

25. R. M. Range and Y. T. Siu, Uniform estimates for the $\bar{\partial}$ equation on domains with piecewise smooth strictly pseudoconvex boundaries, Math. Ann. 206 (1973), 325-354.

26. W. Rudin, Spaces of type $H^{\infty}+C$, Ann. Inst. Fourier (Grenoble) 25 (1975), 99-125.

27. E. M. Stein, Boundary behavior of holomorphic functions of several complex variables, Princeton Univ. Press, Princeton, N.J., 1972.

28. E. L. Stout, $H^{P}$ functions on strongly pseudoconvex domains, Amer. J. Math. 98 (1976), 821-852.

29. U. Venugopalkrishna, Fredholm operators associated with strongly pseudoconvex domains, J. Functional Analysis 9 (1972), 349-373. 
30. K. Yabuta, A remark to a paper of Janas: Toeplitz operators related to certain domains in $\mathbf{C}^{n}$, Studia Math. 62 (1978), 73-74.

Department of Mathematics, Stanford University, Stanford, California 94305

Department of Mathematics, University of California, los Angeles, California 90024 (Current address of S. G. Krantz)

Current address (N. P. Jewell): Medical Computing and Statistics Unit, Medical School, Teviot Place, Edinburgh EH8 9AG, Scotland 